\title{
The association between dietary patterns derived by reduced rank regression and depressive symptoms over time: the Invecchiare in Chianti (InCHIANTI) study
}

\author{
Esther Vermeulen $^{1 *}$, Karien Stronks ${ }^{1}$, Marjolein Visser $^{2,3}$, Ingeborg A. Brouwer ${ }^{2}$, Aart H. Schene ${ }^{4,5,6}$, \\ Roel J. T. Mocking ${ }^{4}$, Marco Colpo ${ }^{7}$, Stefania Bandinelli ${ }^{7}$, Luigi Ferrucci ${ }^{8}$ and Mary Nicolaou ${ }^{1}$ \\ ${ }^{1}$ Department of Public Health, Academic Medical Centre, University of Amsterdam, 1105 AZ Amsterdam, The Netherlands \\ ${ }^{2}$ Department of Health Sciences, EMGO Institute, VU University Amsterdam, 1081 HV Amsterdam, The Netherlands \\ ${ }^{3}$ Department of Nutrition and Dietetics, Internal Medicine, VU University Medical Centre, 1081 HV Amsterdam, The Netherlands \\ ${ }^{4}$ Program for Mood Disorders, Department of Psychiatry, Academic Medical Centre, University of Amsterdam, 1105 AZ \\ Amsterdam, The Netherlands \\ ${ }^{5}$ Department of Psychiatry, Radboud University Medical Centre, 6525 GA Nijmegen, The Netherlands \\ ${ }^{6}$ Donders Institute for Brain, Cognition and Behaviour, Radboud University Nijmegen, 6525 HP Nijmegen, The Netherlands \\ ${ }^{7}$ InCHIANTI Study Group, Azienda Sanitaria Firenze, 50125 Florence, Italy \\ ${ }^{8}$ Longitudinal Studies Section, National Institute on Aging, Baltimore, MD 21224, USA
}

(Submitted 30 September 2015 - Final revision received 12 February 2016 - Accepted 8 March 2016 - First published online 15 April 2016)

\section{Abstract}

This study aimed to identify dietary patterns using reduced rank regression (RRR) and to explore their associations with depressive symptoms over 9 years in the Invecchiare in Chianti study. At baseline, 1362 participants (55.4\% women) aged 18-102 years (mean age 68 (sD 15.5) years) were included in the study. Baseline data collection started in 1998 and was repeated after 3, 6 and 9 years. Dietary intake information was obtained using a country-specific, validated FFQ with 188 food items. For baseline diet, dietary pattern scores in quartiles (Q) were derived using RRR with the nutrients $\mathrm{EPA}+\mathrm{DHA}$, folate, $\mathrm{Mg}$ and $\mathrm{Zn}$ as response variables. Continuous depression scores from the Centre for Epidemiologic Studies Depression (CES-D) scale were used for assessing depressive symptoms. The derived dietary pattern was rich in vegetables, olive oil, grains, fruit, fish and moderate in wine and red and processed meat, and was labelled as 'typical Tuscan dietary pattern'. After full adjustment, an inverse association was observed between this dietary pattern and depressive symptoms at baseline (Q1 $v$. Q4, $B-2 \cdot 77 ; 95 \%$ CI $-4 \cdot 55,-0 \cdot 98$ ). When examining the relationship between the above-mentioned dietary pattern at baseline and depressive symptoms over 9 years, a similar association was found after full adjustment for confounding factors (Q1 v. Q4, $B-1.78 ; 95 \% \mathrm{CI}-3 \cdot 17,-0 \cdot 38)$. A diet rich in vegetables, olive oil, grains, fruits, fish and moderate in wine and red and processed meat was consistently associated with lower CES-D scores over a 9-year period in the Tuscan population.

Key words: Depressive symptoms: Diet: Dietary patterns: Reduced rank regression: Invecchiare in Chianti study

Depression is a common mental disorder and is very common in older persons, especially those affected by medical illness $^{(1,2)}$. Its prevalence and highly recurrent nature make it a major contributor to the global burden of disease. It is estimated to affect more than 350 million people worldwide, and this prevalence is expected to increase in the future ${ }^{(3)}$. Therefore, the prevention of depression is a high priority for public health.

There are indications that diet may play a protective role in the development, progression and treatment of depression and depressive symptoms ${ }^{(4-6)}$. Previous studies have suggested that people suffering from depressive symptoms have higher levels of inflammation and oxidative stress than those without depressive symptoms ${ }^{(7-9)}$. The nutrients EPA and $\mathrm{DHA}^{(10-12)}$, and folate ${ }^{(13-15)}, \mathrm{Mg}^{(16-18)}$ and $\mathrm{Zn}^{(19-21)}$ might have anti-inflammatory properties and appear to be associated with lower depressive symptoms. However, it is difficult to draw conclusions from associations between individual nutrients or foods because people eat meals consisting of several foods with complex combinations of nutrients that are likely to be synergistic or interactive ${ }^{(22)}$.

Abbreviations: CES-D, Centre for Epidemiologic Studies Depression; IADL, instrumental activities of daily living scale; InCHIANTI, Invecchiare in Chianti; Q, quartile; RRR, reduced rank regression.

*Corresponding author: E. Vermeulen, fax +31 20 6972316, email e.vermeulen@amc.uva.nl 
Owing to this fact, studies within several cohorts have examined overall dietary patterns in relation to depressive symptoms with the aim to assess habitual diet as one overall exposure ${ }^{(22-32)}$. The Mediterranean dietary pattern (MDP) is the most commonly investigated dietary pattern and consists of a high ratio of MUFA:SFA, high intakes of fruits and nuts, vegetables, fish, legumes and cereals, moderate intakes of alcohol, milk and dairy products and low intakes of meat and meat products ${ }^{(33)}$. This pattern has been inversely associated with depression in several cohort studies ${ }^{(26,28,32)}$.

To date, most studies have used hypothesis-driven quality scores (a priori) or approaches that are driven by the underlying dietary data (a posteriori) to derive dietary patterns in relation to depression. The a priori approach such as the MDP has the disadvantage that it only includes foods that are hypothesised to have an effect on health and may miss other relevant foods. A posteriori methods such as cluster analysis and principal component analysis have the limitation that they describe the prevailing dietary patterns in a population, and this may not be associated with the disease outcome under study ${ }^{(34)}$.

A hybrid approach, reduced rank regression (RRR), shows complementary value in identifying dietary patterns ${ }^{(35,36)}$. The great advantage of RRR is that it creates a dietary pattern that is associated with the intake of nutrients known to be related to the outcome measure. In this way, the dietary pattern produced is more specific to the outcome of interest ${ }^{(37)}$ in that it identifies the combinations of food intake that explain the most variation in risk for a specific disease ${ }^{(34)}$. Investigating dietary patterns, obtained by RRR, in relation to depressive symptoms using repeated measurements and continuous scores may provide new insights into the relationship between diet and depression.

Therefore, the aim of the present study was to identify dietary patterns using RRR and to explore their associations with depressive symptoms over 9 years in the Invecchiare in Chianti (InCHIANTI) study. Our hypothesis is that participants who adhere to the dietary pattern derived by RRR report fewer depressive symptoms.

\section{Methods}

\section{Participants and study design}

The InCHIANTI study is an ongoing Italian population-based cohort study, which is performed in two sites in Tuscany, Italy (Greve in Chianti and Bagno a Ripoli). Baseline data were collected from 1998 until 2000. At baseline, 1453 people were randomly recruited for the study using a multistage sampling method, and included participants were aged between 20 and 102 years. Participants were excluded from the analysis when baseline data for diet or depressive symptoms were missing ( $n$ 91). In total, 1362 participants were included for the analyses. A home interview was conducted, where participants were asked to answer questionnaires concerning lifestyle, diet and depression. Next, they were invited to the study location for a clinical examination within $21 \mathrm{~d}$ after the home interview. Follow-up data were collected after 3, 6 and 9 years (from 2001-2003, 2004-2006 and 2007-2009, respectively). The study protocol has been described in more detail previously ${ }^{(38)}$.
The ethics committee of the Italian National Institute of Research and Care on Aging approved the study protocol, and informed consent was obtained from all individual participants included.

\section{Depressive symptoms assessment}

The Centre for Epidemiologic Studies Depression (CES-D) scale was used to detect depressive symptoms at baseline and at all follow-ups (after 3, 6 and 9 years) ${ }^{(39)}$. The questionnaire was translated into Italian and was completed by the participants during the home interview ${ }^{(38)}$. The CES-D is a widely used selfreport scale that consists of twenty items (scores range from 0 to 60 points) and has been illustrated to be a valid instrument for identifying depressive symptoms in older adults ${ }^{(40)}$ and in Italians $^{(41)}$. In this study, the continuous CES-D scores were used as outcome measures. CES-D scores were log-transformed when not normally distributed. However, for descriptive purposes, CES-D scores $\geq 20$ points were used to define participants with prevalent depressive symptoms at baseline. This cut-off point was used as it has been shown to have high specificity in identifying depression in Italians ${ }^{(41)}$ and older adults $^{(40)}$ in previous studies. In addition, in order to gain insight into the impact of participants with major depressive symptoms on the studied association, we also conducted sensitivity analysis using the definition major depressive symptoms (CES-D scores $\geq 25$ ). This cut-off point has been previously tested in an older population and has been shown to have satisfactory validity (85\%) and specificity (64\%) in identifying major depressive disorders ${ }^{(42)}$.

\section{Dietary pattern assessment}

Usual dietary intake information was obtained using a countryspecific validated FFQ of North-Central Italy from the European Prospective Investigation into Cancer and Nutrition study containing 248 questions with 188 different food items ${ }^{(43,44)}$. The validation procedure for the FFQ has been described elsewhere $^{(45)}$. Baseline dietary patterns were identified using RRR, which defines linear combinations of food groups (so called predictors) that maximally explain the variation in a set of response variables. These response variables were considered intermediate markers of depressive symptoms and included intakes of fish fatty acids EPA + DHA $(g / d)$, folate $(\mu \mathrm{g} / \mathrm{d}), \mathrm{Mg}$ $(\mathrm{mg} / \mathrm{d})$ and $\mathrm{Zn}(\mathrm{mg} / \mathrm{d})$ from the FFQ. EPA + DHA were chosen instead of total $n-3$ fatty acids because EPA + DHA only contribute a small portion to total $n-3$ fatty acids intake. A maximum of four factors can be extracted from RRR because we used four response variables, and the successive extracted scores are uncorrelated because the eigenvectors are always orthogonal. The RRR method (including the SAS code) has been described in detail by Hoffman et al. ${ }^{(36)}$. As predictors, thirty-one food groups were identified from the FFQ according to their nutritional composition and usual intake based on the Italian National Food Consumption Data in Italian adults (Table 1). Response variables were log-transformed when not normally distributed. Primarily, continuous factor scores were used for all analyses. However, to make factor scores more interpretable, 
Table 1. Food groups from the FFQ for identification of dietary patterns according to their nutritional composition and usual intake in the Invecchiare in Chianti study, Italy

\begin{tabular}{|c|c|c|}
\hline No. & Groups & Foods \\
\hline 1 & Red meat & Beef, veal, pork, mutton, lamb, horse \\
\hline 2 & Processed meat & Processed meat, offals \\
\hline 3 & Poultry & Chicken, turkey \\
\hline 4 & Wild & Rabbit, game \\
\hline 5 & Fish & Fish \\
\hline 6 & Crustaceans/molluscs & Crustaceans, molluscs \\
\hline 7 & Eggs & Eggs \\
\hline 8 & Margarine & Margarine \\
\hline 9 & Other fat & Other animal fat, butter, unclassified oil or fat, other vegetable oils \\
\hline 10 & Olive oil & Olive oil \\
\hline 11 & Dairy products & Yogurt, milk, cheese (including fresh cheese) \\
\hline 12 & Fruit & Fruit \\
\hline 13 & Nuts and seeds & Nuts and seeds \\
\hline 14 & Fruiting vegetables & Tomatoes, other fruiting vegetables \\
\hline 15 & Leafy vegetables & Leafy vegetables \\
\hline 16 & Other vegetables & $\begin{array}{l}\text { Mixed vegetables, mixed salad, onion, garlic, root vegetables, cabbage, } \\
\text { mushroom, stalk vegetables, sprouts }\end{array}$ \\
\hline 17 & Legumes & Legumes \\
\hline 18 & Potatoes & Potatoes \\
\hline 19 & Bread & Bread \\
\hline 20 & Cereals & $\begin{array}{l}\text { Pasta and other grains, crisp bread, rusks, crackers, breadsticks, dry cakes, } \\
\text { pastry (puff, crust, plain) }\end{array}$ \\
\hline 21 & Rice & Rice \\
\hline 22 & Wine & Wine \\
\hline 23 & Other alcoholic drinks & Spirits, brandy, sherry, vermouth, port, beer, cider \\
\hline 24 & Coffee, tea & Coffee, tea \\
\hline 25 & Sugar-sweetened beverages & Carbonate/soft/isotonic drinks, fruit and vegetable juice \\
\hline 26 & Chocolates and sweets & Chocolate confections, non-chocolate confections \\
\hline 27 & Sweet snacks & Ice cream, cake, pie, pastry, pudding \\
\hline 28 & Sugar, honey, jam & Sugar, honey, jam \\
\hline 29 & $\begin{array}{l}\text { Mayonnaise and other } \\
\text { creamy sauces }\end{array}$ & Mayonnaise and similar sauces \\
\hline 30 & $\begin{array}{l}\text { Other sauces (including } \\
\quad \text { tomato sauce) }\end{array}$ & Unclassified other sauces, tomato sauce \\
\hline 31 & Soups & Bouillon, other soups \\
\hline
\end{tabular}

continuous scores were transformed into quartiles, with higher quartiles indicating better adherence to a dietary pattern represented by the nutrients used as response variables.

\section{Covariates}

Models were adjusted for several covariates that were determined to be potential confounding factors: socio-demographic variables included age (in years), sex, education (in years), marital status (never married, married, widowed/divorced) and socio-economic status (SES) (is your current income adequate for your needs: adequate, barely adequate, inadequate). Behavioural variables included the instrumental activities of daily living scale (IADL) (scores: 0-8), the activities of daily living index (ADL) (scores: 0-6), smoking status (never smoker, former smoker, current smoker), alcohol intake (g/d), BMI $\left(\mathrm{kg} / \mathrm{m}^{2}\right)$, waist circumference $(\mathrm{cm})$, physical activity level over the last year (sedentary (hardly any physical activity and mostly sitting/some walking), light (light exercise 2-4h/week), moderate to intense (light $>4 \mathrm{~h} /$ week, moderate $>3 \mathrm{~h} /$ week or intense walks many times per week)) and energy intake $(\mathrm{kJ} / \mathrm{d})$. Health status variables included use of antidepressants (yes or no), hypertension (self-reported and via blood pressure tests) (yes or no), diabetes mellitus type 2 (blood glucose $>126$ $\mathrm{mg} / \mathrm{dl}$ ) (yes or no) and CVD (yes or no).

\section{Statistical analyses}

Frequencies and descriptive statistics were used for categorical (\%) and continuous (mean values, standard deviations) variables, respectively, to compare subject characteristics with the presence or absence of depressive symptoms (CES-D scores of $\geq 20$ ). Normal distribution was checked with QQ plots and the Shapiro-Wilk test.

Cross-sectional association between diet and depression. In total, 1362 participants were included in the analyses. Univariable linear regression analyses were performed to test all potential confounding factors. BMI, waist circumference, ADL, hypertension, diabetes and CVD were not included as confounding factors for further analyses because they did not significantly change the association under study $(\beta<10 \%$ change). Subsequently, multivariable linear regression models with regression coefficients and 95\% CI were used to identify cross-sectional associations between dietary patterns and depressive symptoms. Crude analysis was performed followed 
by adjustment for age, sex, education in years, marital status, SES, IADL, smoking status, alcohol intake, physical activity, energy intake and use of antidepressants.

Prospective association between diet at baseline and depressive symptoms over 9 years. For the analysis, we included participants with at least two measurements of CES-D scores, which left us with 1165 participants. Linear mixed models fit by restricted maximum likelihood (REML) were used, with lowest $v$. highest quartiles of dietary pattern scores at baseline as exposure and repeated measurements of continuous CES-D scores over a period of 9 years as the main outcome. We used linear mixed models, because this method accounts for the dependency of repeated measurements obtained from the same participant over time. In addition, mixed models allow the use of data from participants lost to follow-up. The models initially included only random intercepts; however, random slopes for covariates that may change over time were added to the model and tested for significant improvement in fit (using likelihood ratio tests $(P<0.05))$. Ultimately, the random intercept model was used because it demonstrated the best fit compared with models with random slopes. The linear mixed models included a fixed factor for time. This time variable combines the CES-D scores of all four time periods (baseline and after 3, 6 and 9 years of follow-up) and represents the CES-D scores over time. Other fixed factors included were age, sex, education in years, marital status, SES, IADL, smoking status, alcohol intake, physical activity, energy intake and use of antidepressants at baseline. We did not adjust for CES-D scores at baseline in order to avoid taking away part of the time effect. To further explore the influence of age on the association with depression, all analyses were repeated and models were stratified by age ( $<65$ years, $\geq 65$ years).

Sensitivity analyses. In order to gain insight into the impact of participants with major depressive symptoms on the studied association, we also conducted sensitivity analysis by repeating mixed models and by solely including participants with major depressive symptoms (CES-D scores $\geq 25$ ) at any time point. Finally, participants who dropped out or who missed one or more measurements during the 9-year follow-up period were excluded from the analyses. Analyses were conducted using SPSS version 21 , except for the dietary pattern analyses, which were performed with SAS version 9.3.

\section{Results}

\section{Subject characteristics}

At baseline, 1362 participants were analysed, of whom 264 participants (75\% women) were characterised with depressive symptoms according to the CES-D questionnaire when a cut-off point of $\geq 20$ was used. Baseline characteristics of the study participants according to depressive symptoms are presented in Table 2. On average, participants who reported depressive symptoms were older, were less educated, were more likely to be widowed, had higher IADL scores, had lower energy intake levels and were physically less active compared with participants without depressive symptoms.

\section{Dietary patterns}

In total, RRR extracted four factors, which in total explained $24 \%$ of the variation in the food groups and $84 \%$ of the variation in the response variables. Subsequent analyses were performed with quartiles of the first factor obtained by RRR, because it explained the highest amount of variation in food groups and response variables and all nutrients were correlated more or less on a similar level to the dietary pattern, whereas the remaining factor scores only represented one particular nutrient (Table 3). The second factor score represented a diet high in $\mathrm{EPA}+\mathrm{DHA}$, the third factor score showed foods high in folate and the fourth factor score comprised a diet high in $\mathrm{Mg}$. To identify the more important food groups, a cut-off point of $0 \cdot 15$ was used $^{(46)}$. However, because all food groups are included in the overall score for the dietary pattern per individual, all food groups contribute to some extent to the dietary pattern with RRR. When using this cut-off point, the pattern from the first factor represented a diet characterised by fish, olive oil, several vegetables, fruit, potatoes, cereals, eggs, wine, red and processed meat, and other sauces (particularly tomato sauce) (Table 3). This dietary pattern reflects a typical Tuscan diet and was therefore labelled as 'typical Tuscan dietary pattern'.

\section{Cross-sectional analyses}

In Table 4, the association is shown between the typical Tuscan dietary pattern and depressive symptoms at baseline. Participants with stronger adherence to this dietary pattern reported fewer depressive symptoms (Q1 $v$. Q4, model 1: $B-5 \cdot 60 ; 95 \%$ CI $-6 \cdot 84,-4 \cdot 37)$. When adjusting for demographic variables, the association was weakened but remained statistically significant (Q1 v. Q4, model 2: $B-2 \cdot 18 ; 95 \%$ CI $-3 \cdot 49,-0 \cdot 87$ ). The association became stronger (Q1 $v$. Q4, model 3: $B-2 \cdot 74 ; 95 \%$ CI $-4.53,-0.95)$ after additional adjustment for behavioural variables. Additionally adjusting for antidepressant use did not change the association (Q1 $v$. Q4, model 4: $B-2 \cdot 77$; $95 \%$ CI $-4.55,-0 \cdot 98)$. When repeating the regression analyses with continuous dietary pattern scores as exposure, similar results were found (Table 4).

\section{Prospective analyses}

Table 5 reveals the association between the typical Tuscan dietary pattern at baseline and depressive symptoms over the 9-year follow-up period. Participants in the highest quartile compared with the lowest quartile of the typical Tuscan dietary pattern at baseline had lower CES-D scores during follow-up (Q1 v. Q4, model 1: B -5.16; 95\% CI -6.22, -4.10). After adjustment for demographic variables, the association became considerably weaker (Q1 $v$. Q4, model 2: $B-0 \cdot 95 ; 95 \%$ $\mathrm{CI}-2 \cdot 02,0 \cdot 12)$. After additional adjustment for the behavioural variables, the association became stronger again (Q1 v. Q4, model 3: $B-1 \cdot 70 ; 95 \% \mathrm{CI}-3 \cdot 11,-0 \cdot 29)$ and did not change with adjustment for antidepressant use (Q1 $v$. Q4, model 4: $B-1 \cdot 78$; 
Table 2. Baseline characteristics of study participants according to depressive symptoms in the Invecchiare in Chianti study, Italy* (Mean values and standard deviations; percentages)

\begin{tabular}{|c|c|c|c|c|c|c|}
\hline \multirow[b]{2}{*}{ Characteristics } & \multicolumn{2}{|c|}{ Total sample (n 1362) } & \multicolumn{2}{|c|}{ No depressive symptoms ( $n$ 1098) } & \multicolumn{2}{|c|}{ Depressive symptoms ( $n$ 264) } \\
\hline & Mean & SD & Mean & SD & Mean & SD \\
\hline \multicolumn{7}{|l|}{ Socio-demographic variables } \\
\hline Age (years) & $67 \cdot 9$ & $15 \cdot 5$ & $66 \cdot 7$ & $15 \cdot 6$ & $73 \cdot 1$ & $13 \cdot 8$ \\
\hline Female (\%) & \multicolumn{2}{|c|}{$55 \cdot 4$} & \multicolumn{2}{|c|}{$50 \cdot 6$} & \multicolumn{2}{|c|}{$75 \cdot 0$} \\
\hline Education (years) & $6 \cdot 4$ & $4 \cdot 2$ & $6 \cdot 8$ & $4 \cdot 2$ & $5 \cdot 7$ & $4 \cdot 0$ \\
\hline \multicolumn{7}{|l|}{ Marital status (\%) } \\
\hline Never married & \multicolumn{2}{|c|}{11.9} & \multicolumn{2}{|c|}{$12 \cdot 1$} & \multicolumn{2}{|c|}{11.0} \\
\hline Married & \multicolumn{2}{|c|}{$62 \cdot 6$} & \multicolumn{2}{|c|}{$66 \cdot 2$} & \multicolumn{2}{|c|}{$47 \cdot 3$} \\
\hline Widower/divorced & \multicolumn{2}{|c|}{$25 \cdot 6$} & \multicolumn{2}{|c|}{21.7} & \multicolumn{2}{|c|}{$41 \cdot 7$} \\
\hline \multicolumn{7}{|l|}{ Socio-economic status (\%) } \\
\hline Adequate & \multicolumn{2}{|c|}{$35 \cdot 0$} & \multicolumn{2}{|c|}{$37 \cdot 3$} & \multicolumn{2}{|c|}{$25 \cdot 0$} \\
\hline Barely adequate & \multicolumn{2}{|c|}{$56 \cdot 1$} & \multicolumn{2}{|c|}{$55 \cdot 6$} & \multicolumn{2}{|c|}{$58 \cdot 1$} \\
\hline Inadequate & \multicolumn{2}{|c|}{8.9} & \multicolumn{2}{|c|}{$7 \cdot 0$} & \multicolumn{2}{|c|}{$16 \cdot 9$} \\
\hline Behavioural variables & & & & & & \\
\hline IADL scores $(0-8)$ & 0.52 & 1.41 & 0.33 & $1 \cdot 12$ & $1 \cdot 29$ & 2.08 \\
\hline ADL scores (0-6) & 0.08 & 0.48 & 0.04 & 0.33 & $0 \cdot 26$ & 0.83 \\
\hline Smoking status (\%) & & & & & & \\
\hline Never smoker & & & & & & \\
\hline Former smoker & & & & & & \\
\hline Current smoker & & & & & & \\
\hline Alcohol intake (g/d) & $14 \cdot 8$ & $19 \cdot 8$ & $16 \cdot 1$ & $20 \cdot 5$ & $9 \cdot 1$ & $15 \cdot 5$ \\
\hline Physical activity (\%) & & & & & & \\
\hline Sedentary & & & & & & \\
\hline Light & & & & & & \\
\hline Moderate to intense & & & & & & \\
\hline $\mathrm{BMI}\left(\mathrm{kg} / \mathrm{m}^{2}\right)$ & $27 \cdot 2$ & $4 \cdot 2$ & $27 \cdot 2$ & $4 \cdot 6$ & $27 \cdot 2$ & $4 \cdot 6$ \\
\hline Waist circumference (cm) & $91 \cdot 3$ & $11 \cdot 1$ & 91.5 & 10.9 & 90.6 & $12 \cdot 0$ \\
\hline Energy intake $(\mathrm{kJ} / \mathrm{d})$ & 8405.4 & 2577.8 & $8614 \cdot 8$ & $2577 \cdot 8$ & $7533 \cdot 3$ & $2392 \cdot 3$ \\
\hline Health status variables & & & & & & \\
\hline CES-D score & $12 \cdot 3$ & $8 \cdot 5$ & $8 \cdot 9$ & 4.9 & $26 \cdot 2$ & $5 \cdot 5$ \\
\hline Diabetes (\%) & & & & & & \\
\hline Hypertension (\%) & & & & & & \\
\hline CVD (\%) & & & & & & \\
\hline Use of antidepressants (\%) & & & & & & \\
\hline
\end{tabular}

IADL, instrumental activities of daily living scale; ADL, activities of daily living index; CES-D, Centre for Epidemiologic Studies Depression. * Depressive symptoms are defined as CES-D scores of $\geq 20$ points.

$95 \%$ CI $-3 \cdot 17,-0 \cdot 38)$. No differences in the association were observed when analysing mixed models with the continuous dietary pattern scores (Table 5).

After stratifying for age ( $<65$ years and $\geq 65$ years), participants $<65$ years revealed stronger associations in the fully adjusted models (Q1 $v$. Q4, model 4: $B-2 \cdot 96 ; 95 \%$ CI $-5.43,-0.48)$ compared with the $\geq 65$ years age group (Q1 $v$. Q4, model 4: $B-1 \cdot 02 ; 95 \%$ CI $-2 \cdot 64,0 \cdot 60$ ). Although stronger associations were found in the younger age group, results were consistent in both groups - that is, higher adherence to the typical Tuscan dietary pattern was associated with lower depressive symptoms.

\section{Sensitivity analyses}

For testing the association between diet and major depressive symptoms, the continuous dietary pattern score was used instead of quartiles because of higher power. In participants with major depressive symptoms at any follow-up period ( $n$ 323), the association after full adjustment was quite similar as compared with the analysis that included all participants $(B-0.71 ; 95 \% \mathrm{CI}-1.38$, $-0.04, P=0.04)$. Finally, complete data were available for depressive symptoms at all time points for 722 participants. In this sub-group, the observed association was stronger after full adjustment for confounding factors and was statistically significant (Q1 v. Q4: $B-1 \cdot 82 ; 95 \% \mathrm{CI}-3 \cdot 46,-0 \cdot 17, P=0 \cdot 03$ ).

\section{Discussion}

A diet typically consumed by the Tuscan population - high in vegetables, olive oil, fish, fruits, grains, potatoes, (tomato) sauces, eggs and moderate in wine, red meat and processed meat - was consistently associated with lower depressive symptoms over time in the InCHIANTI cohort. The association between the typical Tuscan dietary pattern and repeated measures of depressive symptoms attenuated after adjustment for confounding factors, but remained statistically significant.

Using RRR to identify dietary patterns and the prospective design of the study with its repeated assessment of depressive symptoms are major strengths of this study. In addition, our study is unique in using continuous scores for depressive symptoms instead of using a cut-off point. This is an advantage because continuous scores are more likely to reflect reality 
Table 3. Overview of food groups with factor loadings $\geq 0.15$ as the cut-off point derived by reduced rank regression with the explained variation and correlation coefficients in the Invecchiare in Chianti study, Italy ( $n$ 1362)

\begin{tabular}{|c|c|c|c|c|c|c|c|}
\hline Factor 1 & Load & Factor 2 & Load & Factor 3 & Load & Factor 4 & Load \\
\hline \multicolumn{8}{|l|}{ Positive loadings } \\
\hline Olive oil & 0.36 & Sweet snacks & 0.43 & Leafy vegetables & 0.41 & Wine & 0.44 \\
\hline Cereals & 0.33 & Fish & 0.37 & Other vegetables & 0.38 & Fish & 0.28 \\
\hline Red meat & 0.28 & Processed meat & 0.31 & Fruiting vegetables & 0.36 & Dairy products & 0.20 \\
\hline Processed meat & 0.27 & Crustaceans and molluscs & 0.25 & Fish & 0.31 & Chocolates \& sweets & 0.20 \\
\hline Other sauces & 0.27 & & & Olive oil & 0.20 & & \\
\hline Bread & 0.24 & & & & & & \\
\hline Leafy vegetables & 0.24 & & & & & & \\
\hline Fruiting vegetables & 0.23 & & & & & & \\
\hline Other vegetables & 0.22 & & & & & & \\
\hline Potatoes & 0.21 & & & & & & \\
\hline Fish & $0 \cdot 18$ & & & & & & \\
\hline Fruit & $0 \cdot 17$ & & & & & & \\
\hline Wine & 0.15 & & & & & & \\
\hline Eggs & 0.15 & & & & & & \\
\hline \multicolumn{8}{|l|}{ Negative loadings } \\
\hline & & Soups & -0.32 & Red meat & -0.34 & Red meat & -0.46 \\
\hline & & Fruit & $-0 \cdot 28$ & Dairy products & $-0 \cdot 21$ & Processed meat & -0.26 \\
\hline & & Olive oil & -0.25 & Other sauces & -0.21 & Other vegetables & -0.23 \\
\hline & & Fruiting vegetables & $-0 \cdot 22$ & Cereals & -0.20 & Fruiting vegetables & -0.22 \\
\hline & & Other vegetables & -0.18 & & & Leafy vegetables & -0.21 \\
\hline \multicolumn{8}{|l|}{ Explained variation } \\
\hline Food groups & $11 \%$ & & $4 \%$ & & $5 \%$ & & $3 \%$ \\
\hline Response variables & $66 \%$ & & $10 \%$ & & $5 \%$ & & $2 \%$ \\
\hline \multicolumn{8}{|c|}{ Pearson's correlation coefficients } \\
\hline $\mathrm{EPA}+\mathrm{DHA}$ & 0.34 & & 0.88 & & 0.30 & & 0.18 \\
\hline Folate & 0.51 & & -0.35 & & 0.69 & & -0.37 \\
\hline $\mathrm{Mg}$ & 0.56 & & -0.31 & & $-0 \cdot 18$ & & 0.75 \\
\hline $\mathrm{Zn}$ & 0.55 & & 0.11 & & -0.64 & & -0.52 \\
\hline
\end{tabular}

Table 4. Cross-sectional association between extreme quartiles of the typical Tuscan dietary pattern and continuous Centre for Epidemiologic Studies Depression scores using multivariable linear regression in the Invecchiare in Chianti study, Italy

(Regression coefficients and 95\% confidence intervals; $n$ 1362)

\begin{tabular}{|c|c|c|c|}
\hline Variable adjustment & Regression coefficient & $95 \% \mathrm{Cl}$ & $P$ \\
\hline \multicolumn{4}{|c|}{ Quartile $1 \mathrm{v}$. quartile $4^{*}$} \\
\hline Model $1 \dagger$ & $-5 \cdot 603$ & $-6.835,-4.371$ & 0.000 \\
\hline Model $2 \ddagger$ & $-2 \cdot 179$ & $-3.490,-0.869$ & 0.001 \\
\hline Model $3 \S$ & $-2 \cdot 738$ & $-4.527,-0.950$ & 0.003 \\
\hline Model $4 \|$ & $-2 \cdot 765$ & $-4.548,-0.982$ & 0.00 \\
\hline \multicolumn{4}{|c|}{ Continuous dietary pattern scores } \\
\hline Model $1 \dagger$ & -1.302 & $-1.572,-1.032$ & 0.00 \\
\hline Model $2 \ddagger$ & -0.498 & $-0.799,-0.198$ & 0.00 \\
\hline Model $3 \S$ & -1.008 & $-1.491,-0.526$ & 0.00 \\
\hline Model 4\| & -0.991 & $-1.473,-0.510$ & 0.00 \\
\hline
\end{tabular}

* Quartile $1=-1.84$ (sD 0.60), quartile $4=2.25$ (SD 1.15).

† Model 1: crude model.

¥ Model 2: adjusted for model 1 and for sex, age, marital status, education in years and socio-economic status.

§ Model 3: adjusted for model 2 and for instrumental activities of daily living scale, smoking status, physical activity, alcohol intake and energy intake.

II Model 4: adjusted for model 3 and for antidepressant use.

compared with using a cut-off point for depressive symptoms, as the latter could be considered arbitrary. It might be argued that interpreting continuous CES-D scores is difficult; thus, we conducted sensitivity analysis by additionally analysing participants with major depressive symptoms and the results were consistent in this group - a typical Tuscan dietary pattern is associated with lower CES-D scores.
Table 5. Prospective association between extreme quartiles of the typical Tuscan dietary pattern at baseline and continuous Centre for Epidemiologic Studies Depression scores over time (at baseline and after 3, 6 and 9 years of follow-up) using linear mixed models in the Invecchiare in Chianti study, Italy

(Regression coefficients and $95 \%$ confidence intervals; $n$ 1165)

\begin{tabular}{|c|c|c|c|}
\hline Variable adjustment & Regression coefficient & $95 \% \mathrm{Cl}$ & $P$ \\
\hline \multicolumn{4}{|c|}{ Quartile 1 v. quartile $4^{*}$} \\
\hline Model $1 \dagger$ & $-5 \cdot 155$ & $-6 \cdot 216,-4.095$ & 0.000 \\
\hline Model $2 \ddagger$ & -0.954 & $-2.023,0.115$ & 0.080 \\
\hline Model $3 \S$ & $-1 \cdot 700$ & $-3.106,-0.294$ & 0.018 \\
\hline Model 4\| & $-1 \cdot 778$ & $-3.171,-0.383$ & 0.013 \\
\hline \multicolumn{4}{|c|}{ Continuous dietary pattern scores } \\
\hline Model $1 \dagger$ & -1.302 & $-1.572,-1.032$ & 0.000 \\
\hline Model $2 \ddagger$ & -0.498 & $-0.799,-0.198$ & 0.001 \\
\hline Model 3§ & -0.704 & $-1.084,-0.325$ & 0.000 \\
\hline Model 4\| & -0.696 & $-1.072,-0.319$ & 0.000 \\
\hline
\end{tabular}

* Quartile $1=-1.74$ (sD 0.58), quartile $4=2.35$ (sD 1.17).

† Model 1: crude model.

‡ Model 2: adjusted for model 1 and for sex, age, marital status, education in years and socio-economic status.

$\S$ Model 3: adjusted for model 2 and for instrumental activities of daily living scale, smoking status, physical activity, alcohol intake and energy intake.

\| Model 4: adjusted for model 3 and for antidepressant use.

A limitation of the current study is that only baseline dietary pattern was included. This means that participants who developed depressive symptoms during follow-up could have altered their diet and we were not able to capture this. We tested for interaction between baseline diet and time in relation to depressive symptoms, and found it to be not significant, 
implying that baseline diet remained stable over time. In addition, it might be that baseline diet was influenced by earlier cases of depressive symptoms and that reverse causality is present. However, in the sensitivity analyses, where we stratified on the basis of severity in CES-D scores, the association between the typical Tuscan dietary pattern and depressive symptoms remained stable. Furthermore, using a self-report memory-based method to obtain dietary information is considered as a limitation. The population under study mainly consisted of older individuals and it could be that imprecisions in dietary recall, due to poor cognitive functioning, attenuated the association under study. However, after excluding participants with poor cognitive function (Mini-Mental State Examination (MMSE) scores <24), the association remained unchanged (data not shown). The self-reported subjective CES-D questionnaire that was used for defining depressive symptoms may also be a limitation because it relies on self-reported answers without a clinical diagnosis of depression. However, the questionnaire has been validated extensively and shows good validity ${ }^{(40,41)}$. Finally, residual confounding cannot be ruled out despite adjustment. Although we adjusted for a number of chronic diseases (hypertension, diabetes, CVD and IADL) in our analyses, this may not cover all conditions that could impact depressive symptoms.

Several cohort studies have investigated the association between dietary patterns in adults and depressive symptoms, using a cut-off point to define depressive symptoms ${ }^{(22,23,25-30,32)}$. Four prospective cohort studies analysed a Mediterranean (like) diet ${ }^{(25,26,28,32)}$, using a priori methods, and found similar results to the prospective results of the present study. A diet high in vegetables, fruits, cereal grains and fish reduced the risk of having depressive symptoms ${ }^{(26,32)}$ or psychological distress ${ }^{(28)}$. Akbaraly et al. ${ }^{(25)}$ observed that women who improved or adhered to a healthy diet had a lower risk of developing recurrent depressive symptoms. Five observational studies derived dietary patterns using a posteriori methods (principal component analysis or factor analysis). Results from these studies were inconsistent. Two of these studies found that higher intakes of fruit, vegetables, fish and poultry lowered the risk of developing depressive symptoms ${ }^{(22,27)}$. In contrast, three other cohort studies did not find a prospective association between a healthy dietary pattern and depressive symptoms ${ }^{(23,29,30)}$. Two studies found a cross-sectional, inverse association between a diet high in vegetables, fruits and olive oil and depressive symptoms; however, when analysed prospectively, the relationship reduced towards the null ${ }^{(29,30)}$. Finally, a cohort study performed in women found no clear association between a healthy dietary pattern (high in fruits, vegetables, fish, poultry and whole grains) and depressive symptoms $^{(23)}$.

Unlike the studies mentioned above that used a priori or a posteriori methods to derive dietary patterns, we created a dietary pattern with food groups high in EPA + DHA, folate, $\mathrm{Mg}$ and $\mathrm{Zn}$, which have been previously inversely associated with depressive symptoms ${ }^{(10-21)}$. Whereas the dietary patterns described in previous studies consisted mainly of vegetables, fish, olive oil, poultry and whole grains, the typical Tuscan dietary pattern also comprised these elements but additionally included red and processed meat, pasta and bread. Red and processed meat (mainly represented by antipasto misto dishes), pasta and bread are typically eaten foods in Tuscany, Italy, because these products are easily accessible and favoured by the population in the Tuscan area, which may explain their important contribution to the dietary pattern derived. However, despite the high factor loadings for red and processed meat, the actual daily intake was quite low (50 and $27 \mathrm{~g} / \mathrm{d}$, respectively), whereas intakes of bread $(109 \mathrm{~g} / \mathrm{d})$ and pasta $(114 \mathrm{~g} / \mathrm{d})$ were higher. Thus, on the basis of our results, it appears that moderate intakes of red and processed meat and higher intakes of pasta and bread fit into a dietary pattern that is consistently associated with lower depressive symptoms. Similar results have been observed by Jacka et $a l{ }^{(47)}$ who found that moderate intakes of red meat (28-57 g/d) does not increase depressive symptoms.

In the sensitivity analysis, we tested whether our findings might be influenced by depression severity. We observed that the inverse association between a typical Tuscan dietary pattern and CES-D scores was similar in participants with major depressive symptoms compared with the observed association of our main analyses, thus confirming our results that a typical Tuscan diet is associated with mild as well as major depressive symptoms.

In addition, the population characteristics and different cultures are not always comparable. It is assumed that not only the quality of the dietary pattern is essential but also the context where people consume their meals might be influential. For example, in the Tuscan population, consuming meals together with friends or family and taking time for meals are considered important. These contextual aspects may be part and parcel of the typical Tuscan dietary pattern, which might reflect a higher quality of life in those consuming it. Overall, future studies should evaluate the role of the social aspects of meal consumption and dietary patterns in relation to depressive symptoms.

\section{Conclusion}

In summary, our results indicate that a typical Tuscan dietary pattern with high intakes of vegetables, olive oil, fish, fruits, cereals and moderate intakes of wine and red and processed meat is consistently associated with lower depressive symptoms over a 9-year period in Italian adults. Our results suggest that diet could be a plausible tool to test in practice to lower depressive symptoms.

\section{Acknowledgements}

The authors thank the participants of the InCHIANTI study for their continuing contributions, all participating departments in the InCHIANTI team and Wim Busschers and Moniek de Goeij for their contribution to the data analysis.

Funding for this research was provided by EU FP7 MooDFOOD Project 'Multi-country cOllaborative project on the rOle of Diet, FOod-related behaviour, and Obesity in the prevention of Depression', grant agreement no. 613598. The InCHIANTI study baseline (1998-2000) was funded by the Italian Ministry of Health (ICS110.1/RF97.71) and in part by the US National 
Institute on Aging, Bethesda, MD (contracts: 236 MD 916413 and 236 MD 821336). The InCHIANTI follow-up 1 (2001-2003) was funded by the US National Institute on Aging (contracts: N.1-AG-1-1 and N.1-AG-1-2111); the InCHIANTI follow-ups two and three (2004-2010) were financed by the US National Institute on Aging (contract: N01-AG-5-0002), supported in part by the Intramural Research Program of the National Institute on Aging, National Institutes of Health.

The authors' contributions are as follows: S. B. and L. F. designed the research and had full access to all the data in the study; E. V., K. S. and M. N. developed the research questions; E. V. analysed the data and had primary responsibility for the final content of the manuscript; E. V. and M. N. drafted the manuscript. All authors critically reviewed the manuscript for important intellectual content and approved the final version of the manuscript. The analyses of the present study were entirely independent, with no industry association.

None of the authors has any conflicts of interest to declare.

\section{References}

1. Beekman AT, Geerlings SW, Deeg DJ, et al. (2002) The natural history of late-life depression: a 6-year prospective study in the community. Arch Gen Psychiatry 59, 605-611.

2. Alexopoulos GS (2005) Depression in the elderly. Lancet $\mathbf{3 6 5}$, 1961-1970.

3. World Health Organization (2012) Fact sheet no. 369 depression. http://www.who.int/mediacentre/factsheets/fs369/ en/ (accessed June 2015).

4. Lopresti AL, Hood SD \& Drummond PD (2013) A review of lifestyle factors that contribute to important pathways associated with major depression: diet, sleep and exercise. $J$ Affect Disord 148, 12-27.

5. Rahe C, Unrath M \& Berger K (2014) Dietary patterns and the risk of depression in adults: a systematic review of observational studies. Eur J Nutr 53, 997-1013.

6. Opie RS, O'Neil A, Itsiopoulos C, et al. (2015) The impact of whole-of-diet interventions on depression and anxiety: a systematic review of randomised controlled trials. Public Health Nutr 18, 2074-2093.

7. Howren MB, Lamkin DM \& Suls J (2009) Associations of depression with C-reactive protein, IL-1, and IL-6: a metaanalysis. Psychosom Med 71, 171-186.

8. Bajpai A, Verma AK, Srivastava M, et al. (2014) Oxidative stress and major depression. J Clin Diagn Res 8, CC04-CC07.

9. Vavakova M, Durackova Z \& Trebaticka J (2015) Markers of oxidative stress and neuroprogression in depression disorder. Oxid Med Cell Longev 2015, 898393.

10. Grosso G, Pajak A, Marventano S, et al. (2014) Role of omega-3 fatty acids in the treatment of depressive disorders: a comprehensive meta-analysis of randomized clinical trials. PLOS ONE 9, e96905.

11. Sublette ME, Ellis SP, Geant AL, et al. (2011) Meta-analysis of the effects of eicosapentaenoic acid (EPA) in clinical trials in depression. J Clin Psychiatry 72, 1577-1584.

12. Bloch MH \& Hannestad J (2012) Omega-3 fatty acids for the treatment of depression: systematic review and meta-analysis. Mol Psychiatry 17, 1272-1282.

13. Sanchez-Villegas A, Doreste J, Schlatter J, et al. (2009) Association between folate, vitamin $\mathrm{B}(6)$ and vitamin $\mathrm{B}(12)$ intake and depression in the SUN cohort study. J Hum Nutr Diet 22, 122-133.

14. Petridou ET, Kousoulis AA, Michelakos T, et al. (2015) Folate and $\mathrm{B}_{12}$ serum levels in association with depression in the aged: a systematic review and meta-analysis. Aging Ment Health (epublication ahead of print version 8 June 2015).

15. Murakami K, Mizoue T, Sasaki S, et al. (2008) Dietary intake of folate, other B vitamins, and omega-3 polyunsaturated fatty acids in relation to depressive symptoms in Japanese adults. Nutrition 24, 140-147.

16. Derom ML, Sayon-Orea C, Martinez-Ortega JM, et al. (2013) Magnesium and depression: a systematic review. Nutr Neurosci 16, 191-206.

17. Jacka FN, Overland S, Stewart R, et al. (2009) Association between magnesium intake and depression and anxiety in community-dwelling adults: the Hordaland Health Study. Aust $N$ Z J Psychiatry 43, 45-52.

18. Tarleton EK \& Littenberg B (2015) Magnesium intake and depression in adults. J Am Board Fam Med 28, 249-256.

19. Vashum KP, McEvoy M, Milton AH, et al. (2014) Dietary zinc is associated with a lower incidence of depression: findings from two Australian cohorts. J Affect Disord 166, 249-257.

20. Maserejian NN, Hall SA \& McKinlay JB (2012) Low dietary or supplemental zinc is associated with depression symptoms among women, but not men, in a population-based epidemiological survey. J Affect Disord 136, 781-788.

21. Miki T, Kochi T, Eguchi M, et al. (2015) Dietary intake of minerals in relation to depressive symptoms in Japanese employees: the Furukawa nutrition and health study. Nutrition 31, 686-690.

22. Akbaraly TN, Brunner EJ, Ferrie JE, et al. (2009) Dietary pattern and depressive symptoms in middle age. Br J Psychiatry 195, 408-413.

23. Chocano-Bedoya PO, O'Reilly EJ, Lucas M, et al. (2013) Prospective study on long-term dietary patterns and incident depression in middle-aged and older women. Am J Clin Nutr 98, 813-820.

24. Lucas M, Chocano-Bedoya P, Shulze MB, et al. (2014) Inflammatory dietary pattern and risk of depression among women. Brain Behav Immun 36, 46-53.

25. Akbaraly TN, Sabia S, Shipley MJ, et al. (2013) Adherence to healthy dietary guidelines and future depressive symptoms: evidence for sex differentials in the Whitehall II study. Am J Clin Nutr 97, 419-427.

26. Sanchez-Villegas A, Delgado-Rodriguez M, Alonso A, et al. (2009) Association of the Mediterranean dietary pattern with the incidence of depression: the Seguimiento Universidad de Navarra/University of Navarra follow-up (SUN) cohort. Arch Gen Psychiatry 66, 1090-1098.

27. Rienks J, Dobson AJ \& Mishra GD (2013) Mediterranean dietary pattern and prevalence and incidence of depressive symptoms in mid-aged women: results from a large community-based prospective study. Eur J Clin Nutr 67 , $75-82$.

28. Hodge A, Almeida OP, English DR, et al. (2013) Patterns of dietary intake and psychological distress in older Australians: benefits not just from a Mediterranean diet. Int Psychogeriatr 25, 456-466.

29. Chan R, Chan D \& Woo J (2014) A prospective cohort study to examine the association between dietary patterns and depressive symptoms in older Chinese people in Hong Kong. PLOS ONE 9, e105760.

30. Le Port A, Gueguen A, Kesse-Guyot E, et al. (2012) Association between dietary patterns and depressive symptoms over time: a 10-year follow-up study of the GAZEL cohort. PLOS ONE 7, e 51593 .

31. Jacka FN, Cherbuin N, Anstey KJ, et al. (2014) Dietary patterns and depressive symptoms over time: examining the relationships with socioeconomic position, health behaviours and cardiovascular risk. PLOS ONE 9, e87657. 
32. Skarupski KA, Tangney CC, Li H, et al. (2013) Mediterranean diet and depressive symptoms among older adults over time. J Nutr Health Aging 17, 441-445.

33. Trichopoulou A, Costacou T, Bamia C, et al. (2003) Adherence to a Mediterranean diet and survival in a Greek population. $N$ Engl J Med 348, 2599-2608.

34. United States Department of Agriculture (2014) A series of systematic reviews on the relationship between dietary patterns and health outcomes. Alexandria, VA: Evidence Analysis Library Division Center for Nutrition Policy and Promotion, US Department of Agriculture.

35. Ocke MC (2013) Evaluation of methodologies for assessing the overall diet: dietary quality scores and dietary pattern analysis. Proc Nutr Soc 72, 191-199.

36. Hoffmann K, Schulze MB, Schienkiewitz A, et al. (2004) Application of a new statistical method to derive dietary patterns in nutritional epidemiology. Am J Epidemiol 159, 935-944.

37. Schulze MB \& Hoffmann K (2006) Methodological approaches to study dietary patterns in relation to risk of coronary heart disease and stroke. Br J Nutr 95, 860-869.

38. Ferrucci L, Bandinelli S, Benvenuti E, et al. (2000) Subsystems contributing to the decline in ability to walk: bridging the gap between epidemiology and geriatric practice in the InCHIANTI study. J Am Geriatr Soc 48, 1618-1625.

39. Radloff L (1977) The CES-D scale: a self report depression scale for research in the general population. Appl Psychol Meas 1, 385-401.

40. Beekman AT, Deeg DJ, Van Limbeek J, et al. (1997) Criterion validity of the Center for Epidemiologic Studies
Depression scale (CES-D): results from a community-based sample of older subjects in The Netherlands. Psychol Med 27, 231-235.

41. Fava GA (1983) Assessing depressive symptoms across cultures: Italian validation of the CES-D self-rating scale. J Clin Psychol 39, 249-251.

42. Haringsma R, Engels GI, Beekman AT \& Spinhoven P (2004) The criterion validity of the Center for Epidemiological Studies Depression Scale (CES-D) in a sample of self-referred elders with depressive symptomatology. Int J Geriatr Psychiatry 19, 558-563.

43. Riboli E, Hunt KJ, Slimani N, et al. (2002) European Prospective Investigation into Cancer and Nutrition (EPIC): study populations and data collection. Public Health Nutr $\mathbf{5}$, 1113-1124

44. Pala V, Sieri S, Palli D, et al. (2003) Diet in the Italian EPIC cohorts: presentation of data and methodological issues. Tumori 89, 594-607.

45. Pisani P, Faggiano F, Krogh V, et al. (1997) Relative validity and reproducibility of a food frequency dietary questionnaire for use in the Italian EPIC centres. Int J Epidemiol 26, Suppl. 1, S152-S160.

46. Miki T, Kochi T, Kuwahara K, et al. (2015) Dietary patterns derived by reduced rank regression (RRR) and depressive symptoms in Japanese employees: the Furukawa nutrition and health study. Psychiatry Res 229, 214-219.

47. Jacka FN PJ, Williams LJ, Mann N, et al. (2012) Red meat consumption and mood and anxiety disorders. Psychother Psychosom 81, 196-198. 